\title{
Analysis of Pump Storaged's Functions at Grid Frequency Control and Contigency Response
}

\author{
Chen Tongfa ${ }^{1,}$, Wan Zhengxi ${ }^{2}$, Yan Lei ${ }^{3}$, Wu Zheng ${ }^{1}$ \\ ${ }^{1}$ Department of Planning, State Grid Xinyuan Holding Company, Beijing, China \\ ${ }^{2}$ East China Tian Huangping Pump Storaged Power Company, Beijing, China \\ ${ }^{3}$ Huilong Branch of State Grid Xinyuan Holding Company, Beijing, China
}

Email address:

187395256@qq.com (Chen Tongfa),wzx0023@126.com (Wan Zhengxi),353102027@qq.com (Yan Lei),dolly_wu@tom.com (Wu Zheng)

${ }^{*}$ Corresponding author

\section{To cite this article:}

Chen Tongfa, Wan Zhengxi, Yan Lei, Wu Zheng. Analysis of Pump Storaged's Functions at Grid Frequency Control and Contigency Response. Science Research. Vol. 7, No. 3, 2019, pp. 38-43. doi: 10.11648/j.sr.20190703.12

Received: June 22, 2019; Accepted: August 4, 2019; Published: October 11, 2019

\begin{abstract}
Frequency stability is one of the crucial indicators of electric power system. Pumped storage hydro (PSH) serves to improve grid security and achieve cost effectiveness and efficiency of overall power system. Frequency regulation is one of PSH's important ancillary services offered to grid. Based on the conventional classifications of frequency regulation, ie., primary frequency regulation (PFR), secondary frequency regulation (SFR) and tertiary frequency regulation (TFR), this paper explores the interreaction process of generation and grid in detailed and proposes two more frequency regulations which are defined as initial frequency regulation (IFR) and $2.5^{\text {th }}$ frequency regulation (contingency frequency regulation, CFR). Therefore the principles and characteristics of five types of frequency regulation are introduced and presented, and performance and economics of various generation units such as hydro, coal-fired, gas, gas combined-cycle and PSH that are engaged in five types of frequency regulation are compared and analyzied in this paper. Especially, the performance of PSH on CFR are illustrated in detail with successful operations of several pump-storage power plants in East China and North China grid under the circumstance of contingency. In one hand PSH's competence on CFR is advantageous compared to other generation units, in the otherhand since the PSH's compensation from frequency regulation is too minor, and more importantly, to avoid mix-up of CFR and contingency reserve which is the core competence of PSH, rhetorical assessment is not proposed on PSH's capability at CFR.
\end{abstract}

Keywords: PSH, Frequency Regulation, Contingency

\section{Introduction}

An essential attribute of electrical system is that electric generation has to match demand in real-time. Either load or generation changes, grid frequency will fluctuate accordingly.

Generally, momentary changes of load comprise of three components: first is random one with time spread less than 10 seconds; Second is pulsed one with time spread between 10 seconds to 3 minutes with larger fluctuation; Third is continuous and cycled components changing slowly with daily and seasonal patterns, are the main proportion of load changes [1]. Plus, momentary outage or disconnected unexpectedly of generation equally resemble in effect demand surges sharply, which are significant events for grid, and distinguished from primary and secondary load changes in conventional view [1]. To be easier to illustrate, this article will take that as a new component and classify the above four load changes as primary shock, secondary shock, tertiary shock and $2.5^{\text {th }}$ shock.

To regulate frequency deviation caused by unmatch of generation and demand, four responses, i.e., Initial frequency regulation (IFR), primary frequency regulation (PFR), secondary frequency regulation (SFR), tertiary frequency regulation (TFR) and contingency frequency regulation (CFR) are presented in this paper.

\section{Responses of Grid Frequency Regulation}

The definition of frequency regulation, is the responses 
both from generation and load when grid experiences impulse. When frequency fluctuates due to unbalance of active power and load, frequency regulation varies in stages and may be categorized sequentially as follows: generator's IFR (coming from mass inertia) and load's power-frequency cooperate effect (the two are fully synchronized) $\rightarrow$ generation's PFR $\rightarrow$ generation's SFR $\rightarrow$ generation's $2.5^{\text {th }}$ frequency regulation under the circumstance of emergency $\rightarrow$ generation's TFR.

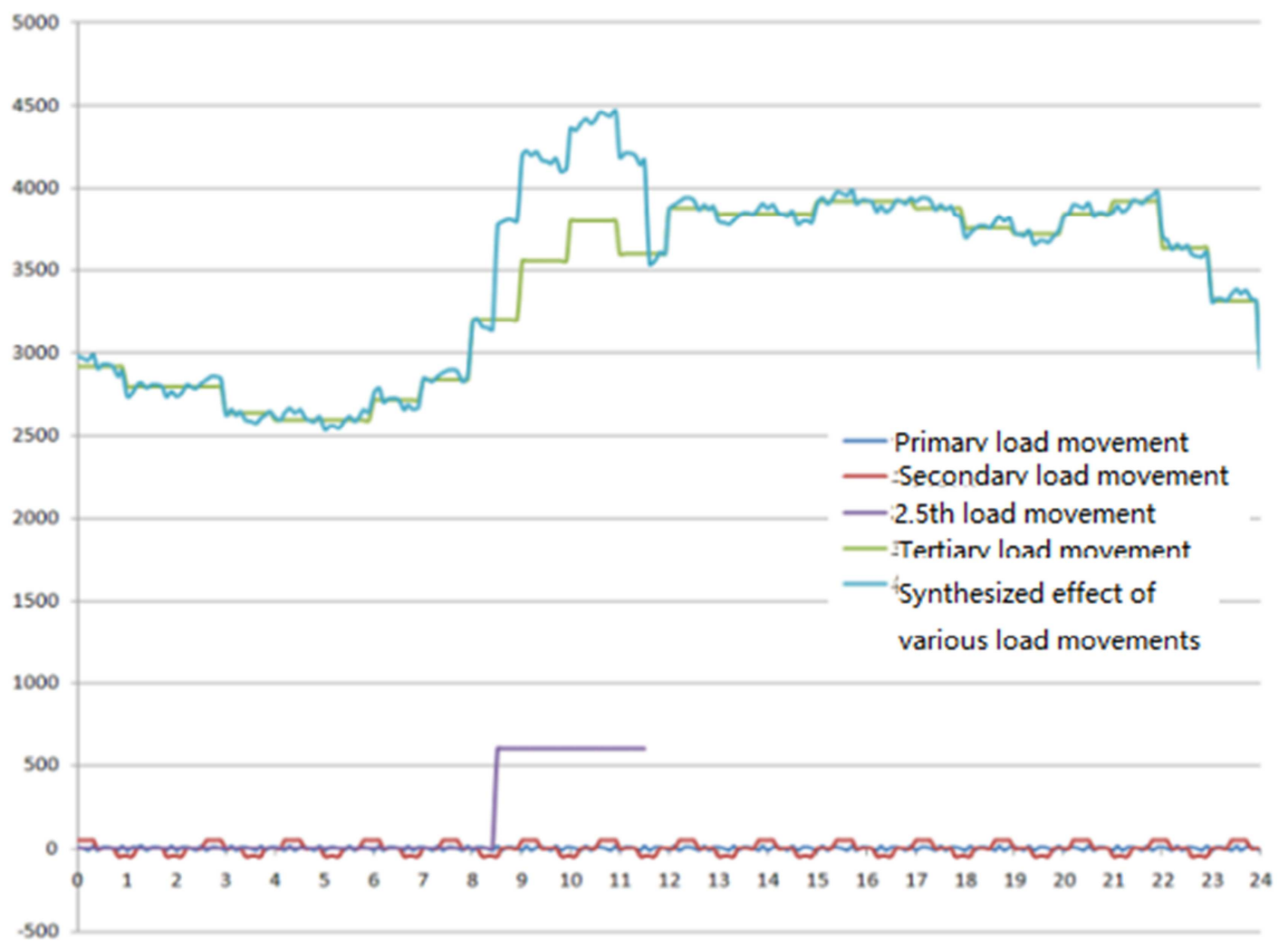

Figure 1. Typical daily load curve.

Note: (a) rotating parts at demand side have mechanical inertia and are parts of IFR. Nonetheless the effect from them is too minor to be taken into account. (b) frequency regulation under the circumstance of contingency is a significant event, but not included in 3 types of conventional frequency regulation. Thus it is defined as " $2.5^{\text {th }}$ frequency regulation" hereafter since its response time is between SFR and TFR.

\subsection{Initial Frequency Regulation (IFR)}

Time interval lies between grid frequency deviating to governor kicking off. Before governor starts to work, frequency deviation is resisted and mitigated by generator's rotating inertia. This is called initial frequency regulation. The following describes the basic attributes of generator's frequency control.

$$
\left.\begin{array}{c}
T_{J} \frac{d \omega}{d t}=M_{m}-M e-D(\omega-1) \\
\frac{d \delta}{d t}=\omega-1
\end{array}\right\}
$$

Where $T_{J}$ refers to inertia constant of generator; 6 is the angel between quadrature axis of rotor and direct axis rotating at synchronous speed; $M_{m}$ is mechanical torque; $M e$ is electromagnetic torque; $\mathrm{D}$ refers to damping coefficient; $\omega$ is angular frequency and $\omega=2 \pi f[10]$.

IFR of generator units can postpone grid fluctuation 1 to 2 seconds and be of great importance for reducing grid frequency deviation [2].

Inertia constant of generator can be calculated in a simpler and direct way as following:

Example\#1: generator's rotating inertia is included in generator's design to avoid run-away speed of turbine too high in the case of load shedding. Weight will be put on rotor if inertia value is too low. Run-away speed is roughly $170 \%$ to $260 \%$ of rated speed [8].

Example \#2: sub-grid with voltage of $110 \mathrm{Kv}$ was disconnected from main grid with voltage of $220 \mathrm{Kv}$ accidentally and temporarily isolated. Generation capacity of the sub-grid tumbled from $44.4 \mathrm{MW}$ to $24.9 \mathrm{MW}$ with the loss of $19.5 \mathrm{MW}$ and $43 \%$ in terms of percentage. According to the calculation of grid frequency, theoretically the frequency in static balance should drop $9 \mathrm{~Hz}$. before primary frequency control functioning. Thanks to IFR, frequency only drooped $1.53 \mathrm{~Hz}$ in a second, i.e., governor was not initiated. If we considered the sheer power-frequency relationship, $2.25 \mathrm{MW}$ of demand rising (or supply cutting) abruptly could lead to $1.53 \mathrm{~Hz}$ reduction instantly [4]. 
Table 1. Typical frequency changing process against time.

\begin{tabular}{llllllllllll}
\hline T $(\mathbf{s})$ & $\mathbf{0}$ & $\mathbf{0 . 1}$ & $\mathbf{0 . 2}$ & $\mathbf{0 . 3}$ & $\mathbf{0 . 4}$ & $\mathbf{0 . 5}$ & $\mathbf{0 . 6}$ & $\mathbf{0 . 7}$ & $\mathbf{0 . 8}$ & $\mathbf{0 . 9}$ & $\mathbf{1}$ \\
\hline $\mathrm{F}(\mathrm{Hz})$ & 49.87 & 49.68 & 49.32 & 49.36 & 49.21 & 49.07 & 48.91 & 48.75 & 48.6 & 48.74 & 48.34 \\
\hline
\end{tabular}

Obviously generation units in operation mode have contributions to IFR. Contribution level is depended on generator's rated capacity instead of operating capacity.

\subsection{Power-frequency Regulation Effect}

Active power will rise or drop to adapt to grid frequency fluctuating accordingly. This relationship can be expressed in per-unit value as following:

$$
K_{L^{*}}=\frac{\Delta P_{L} / P_{L N}}{\Delta f / f_{N}}=\frac{\Delta P_{L^{*}}}{\Delta f_{*}}=K_{L} \frac{f_{N}}{P_{L N}}
$$

Where $\mathrm{K}_{\mathrm{L}}$ refers to power load changes; $\mathrm{K}_{\mathrm{L}^{*}}$ refers to per-unit value of $\mathrm{K}_{\mathrm{L}}$, $\mathrm{P}_{\mathrm{L}}$ refers to overall power load when grid frequency is $f ; \mathrm{f}_{\mathrm{N}}$ is rated frequency; $\mathrm{P}_{\mathrm{LN}}$ refers to overall power load when grid frequency is $\mathrm{f}_{\mathrm{N}}$.

When $\mathrm{K}_{\mathrm{L}^{*}}=1-3$, power load changes $1 \%-3 \%$ for every $1 \%$ of frequency change. $\mathrm{K}_{\mathrm{L}^{*}}$ is not adjustable but rather hinges on load mix and various load properties [3].

Power-frequency regulation effect will take place at the moment of frequency deviation and go through the whole process.

Power-frequency regulation effect can postpone and mitigate grid frequency deviation which is not substantial however [4]. For example, set $\mathrm{KL}^{*}=1.5$ (East China grid), peak load $=400 \mathrm{GW}$, a loss of $240 \mathrm{MW}$ of generation could result in a drop of $0.2 \mathrm{~Hz}$. in the case of excluding the power-frequency regulation effect from generation [5].

\subsection{Primary Frequency Regulation (PFR)}

At the time grid frequency changes, generator's governor will change the input from turbine accordingly. Output of generator will be adjusted to match the demand at a new level and grid frequency is restored into a normal range of deviation. Relationship of generation corresponding to frequency movement is depicted as generator's power \& frequency characteristic.

$$
K_{G^{*}}=\frac{1}{\sigma_{*}}=\frac{-\Delta P_{G^{*}}}{\Delta f_{*}}
$$

$\mathrm{K}_{\mathrm{G}^{*}}$ is factor of generator's power \& frequency static charicteristic; $6 *$ refers to per-unit value of deviation regulation factor for generator; $\Delta f_{*}$ refers to drop of frequency; $\Delta \mathrm{P}_{\mathrm{G}^{*}}$ is addition of generation after $\Delta f_{*}$.

$\mathrm{K}_{\mathrm{G}^{*}}$ is adjustable. Frequency deviation will be less while $\mathrm{K}_{\mathrm{G}^{*}}$ is getting larger. For steam turbine, $6 *=3 \%-5 \%, \mathrm{~K}_{\mathrm{G}^{*}}$ $=16.7-25$; for hydro turbine, $6 *=2 \%-4 \%, \mathrm{~K}_{\mathrm{G}^{*}}=25-50[6]$.

Generator's PFR features prompt reaction to grid frequency fluctuation and is the first mean of active frequency control safeguarding grid reliability. According to IEEE statistics, time interval of primary frequency control is in the order of 10 seconds. North China Grid claims that interval from the time PFR initiating to the time generation and load starting to respond is less than 3 seconds [11].

Since generator's PFR mainly influences the valves of steam turbine rather than combustion system, heat stored in boiler tentatively boost the output of turbine as the valve opens wider. But the chemical energy stored in combustion system remains unchanged, output of turbine will restore to prior level with the heat stored getting less. Thus the PFR of steam turbine does not last long. The time of PFR of steam turbine ranges from $0.5-2$ minutes subject to various generator units with various heat stored.

Only operating units can contribute to PFR. Generator's PFR can not take effect if it is not on-line no matter how fast it can start and ramp. The capability of generator's PFR is depended on its rated capacity, i.e., correlated with $6 \%$ and little with ramp rate. The capability of generator's PFR is basically in the order of following: hydro $<\mathrm{PSH}<$ thermal [7].

\subsection{Secondary Frequency Regulation (SFR)}

SFR is mainly conducted by generator units in charge of grid frequency control. Output of these units can be varied by changing the parameters of governor system and restore the grid frequency to required level.

PFR can not achieve no-deviating regulation because of its deviating regulation and attenuation. No-deviating regulation needs participation of SFR.

Response time of SFR is 1 to 2 minutes which is much longer than PFR due to the energy transformation inside generator units being more complex and taking more time. Slow response of secondary can tackle load fluctuation at minutes or longer level rather than followling the random movements of demand rapidly.

PFR and SFR is illustrated at figure 2.

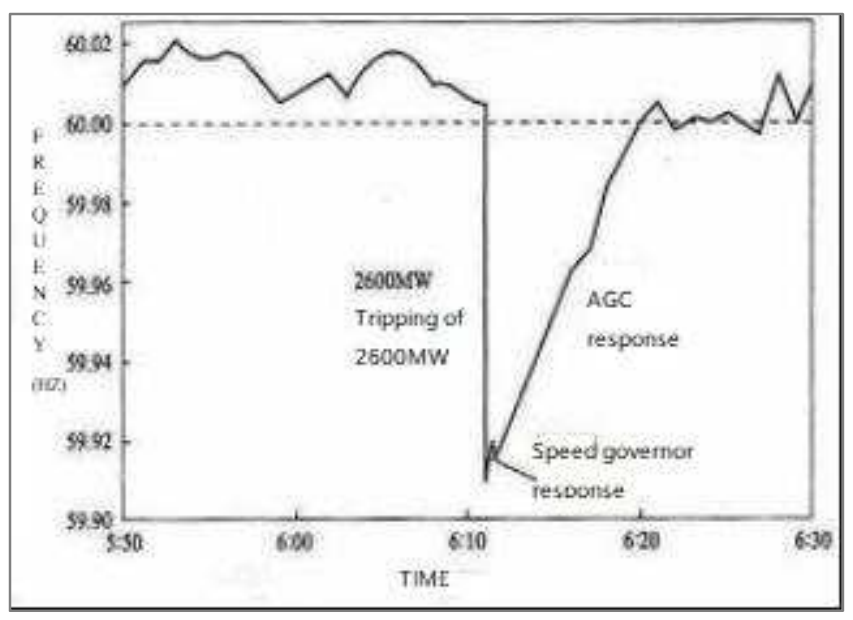

Figure 2. Frequency regulation functioning process. 
Capability of SFR is related to the ramp rate of generator unit. Overall speaking, the ramp rates of coal-fired, gas turbine, gas combined-cycle turbine, hydro and PSH in terms of rated capacity per minute are $1 \%-2 \%, 50 \%, 1 \%-2 \%, 30 \%-50 \%$ and $50 \%$ respectively [9].

\section{5. $2.5^{\text {th }}$ Frequency Regulation (CFR)}

$2.5^{\text {th }}$ shock in grid features huge capacity loss at either generation or demand side and drastic frequency fluctuation. The probability of $2.5^{\text {th }}$ strike occurrence is relatively high. The frequency deviation of more than $0.1 \mathrm{~Hz}$ will be claimed to be emergency accident in terms of East China Grid regulations.

In a bid to tackle $2.5^{\text {th }}$ shock in grid, generation units capable of $2.5^{\text {th }}$ frequency regulation must be of large capacity and high ramp rate, both of which $\mathrm{PSH}$, gas turbine units and hydro all boast.

Generally construction and operation of gas turbine power plant is costly. Hydro power development is constraint by water resource, topography and distantance from load centre. Hydro power should be given priority to undertake $2.5^{\text {th }}$ frequency regulation in the area hydro is available. More over, PSH should be developed where construction cost is affordable. Gas turbine power is the last choice at the absence of PSH.

\subsection{Tertiary Frequency Regulationy (TFR)}

SFR and $2.5^{\text {th }}$ frequency regulation will inevitably push generation units from operating economically, which needs to be taken care of by TFR.

The task of TFR is to achieve balance between generation and load in an economical and efficient way corresponding to daily load movements through combination of generation units with lowest cost of starting up or closing down.

Distinguished from PSR and SFR, TFR not only has to response to real-time demand, but also make arrangements for generation regarding to forecasted demand.

An metaphorical depiction of TFR is peak shaving and valley filling in daily load chart. The cycle of regulation is normally 5-10 minutes.

The outstanding feature of TFR is the enormous capacity of units required to participate in grid, however, the ramp rate of units is not a rigid parameter.

$\mathrm{PSH}$, gas-steam combined-cycle, thermal and hydro with flexible regulation capability are all eligible regardless of operation cost.

\section{Economic Comparison of Above Frequency Regulations}

\subsection{Initial Frequency Regulation (IFR)}

This function is vital for all units without extra cost.

\subsection{Primary Frequency Regulation (PFR) and Secondary Frequency Regulation (SFR)}

PFR is the liable duty of all units on-line.

As for SFR, response and ramp rate of units both are valuable in power market, but the amount of fee payable to frequency regulation is rather small. The proportion of frequency regulation is only $0.62 \%$ of retail price of electricity in PJM, US., and no more than $1 \%-2 \%$ in states grid in Australia [12].

Grid frequency fluctuations caused by primary and secondary demand changing are mostly in the normal range of frequency deviation, and do not lead to serious accident for grid.

\section{3. $2.5^{\text {th }}$ Frequency Regulation (CFR)}

In most case PSH units and gas turbine units are the two types of generation units that are capable of undertaking $2.5^{\text {th }}$ frequency regulation. Besides, only sizable units widely interconnected can be up to the requirements of CFR. Although hydro is eligible for CFR, it is not always applicable because it is susceptible to external factors.

Gas-steam combined-cycle power generation is put forward as the efficiency of energy usage of gas turbine units is as low as $30 \%$. However, with the addition of boiler, the ramping of gas combined-cycle units is as slow as coal-fired units, and thus it does not meet the requirements of CFR either.

Therefore, PSH and gas turbine units are the two alternatives for CFR in grid.

\subsection{Tertiary Frequency Regulation (TFR)}

Generation units undertaking TFR may make profits through buying low and selling high in power market and receiving fixed payments corresponding to capacity. PSH units are regarded as the best ones for undertaking TFR. In the other hand, proportion of PSH to overall installed capacity in grid should be limited since PSH generates $3 \mathrm{Kwh}$ at the cost of $4 \mathrm{Kwh}$ roughly.

\section{Analysis of PSH's Frequency Regulation}

\subsection{Initial Frequency Regulation (IFR)}

PSH makes no difference with hydro and thermal for Initial frequency regulation commonly originated from inertial of mass. However, PSH performs poorly since IFR is only applicable for operating units and PSH operating much less than other units in terms of time.

\subsection{Primary Frequency Regulation (PFR)}

Again, PSH is of no substantial advantage over hydro and thermal units in regard of PFR.

\subsection{Secondary Frequency Regulation (SFR)}

PSH units are competent at SFR for its relatively large capacity and fast ramping. However, operating capacity in grid is generally redundant to engage in SFR. Therefore the demand for PSH is not tense. For example, Shi Sanling PSH tops the list of power plants engaging in frequency 
regulation in North China Grid which is short of hydro. North China Grid requires that Shi sanling PSH turns on $\mathrm{AGC}$ as long as units start up. Time interval of frequency regulation order receiving from grid is longer than 5 minutes. For East China Grid, frequency regulation order is dispatched to PSH units with AGC turning on every 15 minutes. In this case, PSH is undertaking duty of TFR. For Central China Grid, taking Baoquan PSH as an example, its AGC was only put into operation 4 times in 3 years. Baoquan PSH involved no frequency regulation activities since it was commissioned.

\section{4. $2.5^{\text {th }}$ Frequency Regulation (CFR)}

At present, large capacity unit and ultra-high voltage transmission line have been common in grid. Accidental events featuring loss of GWs occur frequently in recent grids.

Besides, many non-accidents came along with features of emergency accident. For example, in 2014 load had changed sharply in the range of $4.5 \mathrm{GW}$ in 20 minutes at around 23:00 for quite a long time in Shandong Grid.

PSH units excel at SFR for its relatively large capacity and fast ramping. Taking Baoquan PSH in central China as an example, it responded to demand of CFR dozen times during year 2013 winter peak times and secured the grid reliability. That is common for other PSH in China. Tong Bai PSH in East China with 4 units and installed capacity of $1200 \mathrm{MW}$, responded to order from East China Grid of CFR during year 2014 summer peak times many times. For example, 2 units $(600 \mathrm{MW})$ started up urgently at the order of grid dispatching centre and engaged in generation for 80 minutes from 21:30-22:20 on 27th, July; 3 units (900MW) were put into generation at the time of $18: 42,19^{\text {th }}$, September 2014 [13].

Thus $2.5^{\text {th }}$ frequency regulation (CFR) is proved to be one of PSH's important functions to secure reliability and enhance flexibility of grid.

Gas units could be an alternative for PHS in regard of CFR. However, the operation cost of gas units is much as twice as that of PSH due to the low rate of energy transforming efficiency (around 30\%). Generally speaking, priority should be given to PSH to undertake CFR as long as the topographical and geological conditions available.

\subsection{Tertiary Frequency Regulation (TFR)}

PSH is capable at TFR due to its large capacity in general. Nonetheless the capability should be compromised since PSH generates $3 \mathrm{kwh}$ at a cost of $4 \mathrm{kwh}$. For each grid with PSH, generation mix changes dynamically and there is an economical combination of PSH and other generations in real-time to response to TFR. A comprehensive computation regarding to cost efficiency of TFR for a specific grid must be done to determine whether PSH should be put into operation.

\section{Conclusions}

A. Frequency regulation mainly refers to PFR and SFR. The cost of both that accounts for in retailing price of electricity is hardly countable. Therefore, the pricing of electricity sales of PSH, and more specifically, the cost of frequency regulation could not be properly reflected in power market or compensated by ancillary services.

B. Contingency reserve is one of PSH's key functions. In one hand, it is the sheer reason for development proposal of $\mathrm{PSH}$; in the other hand, the alternative cost of PSH is rather pricey. Appreciation of Contingency reserve of PSH should cover the whole development process, including project necessity examination in prefeasibility study stage, economical evaluation in feasibility study stage, operation mode and investment recuperation approach after project commissions [14].

C. Overstatement of PSH frequency regulation capability, and misunderstanding of frequency regulation and contingency reserve, could result in weakening of contingency reserve which is the core competence of PSH [15].

D. Coal-fired units are taken as alternative scheme to evaluate PSH cost effectiveness at stage of prefeasibility study for PSH project. In fact, coal-fired units and gas turbine units can be the alternative schemes for peak regulation and contingency reserve. Therefore cost effectiveness of PSH will be more evident and objective.

\section{References}

[1] Suai Junqing, Key technologies applied in extra-large grid dispatching centre, published by China Electric Power Press, Beijing, 2010.

[2] Gao Xiang, Applied technologies for frequency control in modern grid, published by China Electric Power Press, Beijing, 2010.

[3] Xia Daozhi, Analysis of electric power system, published by China Electric Power Press, Beijing, 2010.

[4] Wang Weichao, Zhang Ming, Hu Kun, Operation mode of electric power system, published by China Electric Power Press, Beijing, 2009.

[5] Du Liang, Unleashed process and indicators system of frequency regulation capacity, published by Grid Technology, 2007, 31 (21).

[6] Wang Hezhen, Record analysis of load responses df/da and grid frequency characteristics. published by Grid Technology, 1995, 19 (12).

[7] Yang Jianhua, Steady state analysis and economic operation of electric power system, published by China Electric Power Press, Beijing, 2013.

[8] Cheng Haozhong, Introduction of power quality, published by China Electric Power Press, Beijing, 2013.

[9] Zhang Zhichao, Wang Zengping, Fang Panyu, Strategy study under emergency situation based on frequency and voltage [J], Protection and Control of Power System, 2013, 41 (03): 149-155.

[10] Ed Habtour; Mark Paulus; Abhijit Dasgupta. Modeling Approach for Predicting the Rate of Frequency Change of Notched Beam Exposed to Gaussian Random Excitation [J], Shock and Vibration, 2014. 
[11] Tang J, Liu J, Ponci F. Adaptive load shedding based on combined frequency and voltage stability assessment using synchrophasor measurements [J]. IEEE Transactions on Power Systems. 2013, 28 (2): 2035-2047.

[12] Albu, M.; Dumitrescu, A. M.; Popovici, R., Rate of change of frequency - a power quality descriptor [A]. 2014.

[13] Ying-Yi Hong, Shih-Fan Wei. Multiobjective underfrequency load shedding in an autonomous system using hierarchical genetic algorithms [J]. IEEE Transactions on Power Delivery, 2016, 25 (3): 1355-1362.

[14] CIGRE SCTF 38.02.19. System protection schemes in power networks [R]. 2017.

[15] Yuan Ji Xiu. Security and Stability of Power System [M]. Published by China Electric Power Press, Beijing, 2018. 